\title{
Evaluation of ovarian structures using computerized microtomography
}

\author{
FERNANDA PAULINI ${ }^{1}$, SACHA B. CHAVES ${ }^{2}$, JOSÉ LUIZ J.P. RÔLO ${ }^{1}$, \\ RICARDO B. DE AZEVEDO ${ }^{2}$ and CAROLINA M. LUCCI ${ }^{1}$
}

\author{
${ }^{1}$ Departamento de Ciências Fisiológicas, Instituto de Ciências Biológicas, Universidade de Brasília, \\ Campus Universitário Darcy Ribeiro, Asa Norte, 70910-900 Brasília, DF, Brazil \\ ${ }^{2}$ Departamento de Genética e Morfologia, Instituto de Ciências Biológicas, Universidade de Brasília, \\ Campus Universitário Darcy Ribeiro, Asa Norte, 70910-900 Brasília, DF, Brazil
}

Manuscript received on December 7, 2015; accepted for publication on June 22, 2016.

\begin{abstract}
Visualization and clear understanding of the ovarian structures are important in determining the stage of oestrus, helping to diagnose several pathologies and supporting advances in reproductive technologies. In this research, computerized microtomography (microCT) was used to explore and characterize the ovarian structure of seven mammalian species. Ovaries of rats, female dog, queens, cows, mares, sows and a female donkey were used. After microCT scanning, the same samples were prepared for histologic evaluation, used here as a validation criterion. It was possible to distinguish regions of the cortex and medulla, visualize the morphology and distribution of blood vessels, clearly observe corpus luteum and antral follicles, and visualize oocytes inside some antral follicles. This is the first report using microCT to explore and compare ovarian structures in several domestic mammals. MicroCT revealed great potential for the evaluation of ovarian structures. This research open prospects for the use of computerized tomography (CT) as a noninvasive approach to studying ovarian structures in live animals, which may be especially attractive for scientific study of development of ovarian structures and/or ovarian pathologies in small animals' models.
\end{abstract}

Key words: ovarian follicle, blood vessels, histology, tomography, ovary.

\section{INTRODUCTION}

The ovary is a complex and specialized organ, and visualization and clear understanding of ovarian structures are important in determining the stage of oestrus, helping to diagnose several pathologies and supporting advances in reproductive technologies.

Several imaging techniques have been used to perform ovary evaluation and diagnosis. Currently,

Correspondence to: Carolina Madeira Lucci

E-mail: carollucci@gmail.com the most common tool for in vivo evaluation of human and animal ovaries is ultrasound, which allows the identification of ovarian tumours and cysts (Prater et al. 1988, Vlaisavljevic and Došen 2007). Doppler ultrasound boosted the precision of the differentiation of some malign pathologies that are frequently observed in the ovary and that normally present neovascularization regions (Giordano et al. 2009).

In the 1970's computerized tomography (CT) appeared as a new route for imaging diagnosis. 
Computerized microtomography (microCT) is a lower-scale $\mathrm{CT}$, with even greater precision (Guldberg et al. 2003), developed for research studies. MicroCT has been frequently used to evaluate mineralized tissues, such as bones and teeth (Davis and Wong 1996), as well as brain tissue (Langheinrich et al. 2010), blood vessels (Langheinrich et al. 2004, Barreto et al. 2008, Nebuloni et al. 2014) and lungs (Postnov et al. 2005, Ashton et al. 2014). Concerning the ovaries, CT has only been used to visualize tumours, torsions and a few other pathologies in humans (Sawyer et al. 1985, Foshager and Walsh 1994, Hamm 1994, Saksouk and Johnson 2004, Giordano et al. 2009, Shaaban and Rezvani 2009, Moribata et al. 2015), register morphological aspects in monkeys (Jones et al. 2007, 2010) and tumours in rats (Hariri et al. 2010). Although the contribution of CT to ovarian investigation is still insufficient, the characteristics of microCT are interesting for ovary imaging.

MicroCT is a non-invasive technique that can detect less than $1 \%$ density differences, allowing micrometric evaluation of structures (Bontrager 2003). Although both ultrasonography and CT permit the visualization of ovarian structures, CT allows a three-dimensional (3D) reconstruction of the organ and the visualization of specific sections of interest in much greater detail. The images generated by $\mathrm{CT}$ are comparable in resolution to those of histological sections (Metscher 2009a). However, while high-resolution techniques (i.e. histology and confocal laser scanning microscopy) usually depend on biopsies for tissue evaluation, CT could be used in live individuals (Metscher 2009b). Other features of $\mathrm{CT}$, such as speed, precision and interactivity, reinforce the need to verify the potential of this technique in ovarian evaluation. In these terms, the present study aimed to verify if microCT is suitable to explore and characterize the ovarian structures of seven mammalian species.

\section{MATERIALS AND METHODS}

Whole ovaries from rats (Rattus novergicus) $(\mathrm{n}=2)$, female dogs (Canis lupus familiaris) $(\mathrm{n}=2)$, cows (Bos taurus) $(\mathrm{n}=2)$ and queen (Felis catus domesticus) $(\mathrm{n}=1)$ and hemi-ovaries from sows (Sus domesticus) $(\mathrm{n}=3)$, mares (Equus caballus) $(\mathrm{n}=3)$ and female donkey (Equus africanus asinus) $(n=1)$ were used in this study. All ovaries were collected from adult, healthy and non-pregnant animals. Ovaries from female dogs, queen, mares and female donkey were obtained from animals subjected to elective ovariohysterectomy at a local veterinary clinic/hospital. Ovaries from sows and cows were collected in certified slaughterhouse and the ovaries from rats were collected from an animal euthanized at the University Animal Facility.

All samples were fixed in Carnoy solution $(60 \%$ ethanol, 30\% chloroform and 10\% glacial acetic acid), overnight for hemi-ovaries of mares, female donkey, sows and cows, or for 8 hours for ovaries of female dogs, queen and rats, and then immersed in $70 \%$ ethanol and kept under refrigeration $\left(4^{\circ} \mathrm{C}\right)$.

\section{COMPUTERIZED MICROTOMOGRAPHY}

Before scanning, all ovaries and hemi-ovaries were immersed in a solution of $70 \%$ ethanol and $1 \%$ tincture of iodine $(10 \%)$ in water for 24 hours to obtain contrast. Ovaries were scanned using a Skyscan 1076 MicroCT (Skyscan, Aartselaar, Belgium) at $100 \mathrm{kV}, 100 \mathrm{~mA}, 9 \mu \mathrm{m}$ pixel size, with Al $1 \mathrm{~mm}$ filter, rotation step of $0.3^{\circ}$ and $1 \mu \mathrm{Sv} / \mathrm{h}$ for radiation. Bi-dimensional reconstruction of the images was performed using the NRecon software (V 1.6.9, version 64 bit with GPU acceleration) and three-dimensional reconstruction using CTvox software (V 2.7, version 64 bit) and DataViewer (1.5.0 version 32 bit). All software used was developed by Skyscan (Kontich, Belgium). For calculation of standard x-ray attenuation units (Hounsfield units, HU) a phantom object was used. For this purpose, polypropylene tube of $50 \mathrm{~mL}$ 
volume was filled with deionized water and scanned in the microCT device using the same parameters used for each species scanned. To calculate the mean density value of HU for the water phantom, CT software was used.

\section{HISTOLOGY}

Histology was used as a cross-checking technique, to validate the findings observed in microCT. After scanning, samples were individually transferred to $70 \%$ ethanol solution, dehydrated in ethanol, clarified with xylene, and embedded in paraffin wax (Paraplast, Sigma). From each ovary, 5 sections of $5 \mu \mathrm{m}$ were cut, mounted on microscopic slides and stained with hematoxylin and eosin (HE).

Microscopic slides were evaluated using a light microscope (Leica DM1000, Leica, Wetzlar, Germany); photographs were taken with a digital camera (Leica DFC280, Leica, Wetzlar, Germany) with the aid of Leica Application Suite software (version 2.7.0 R1 Copyright 20032007, Leica Microsystems, Switzerland). Images of all microscopic fields were captured at $4 \mathrm{x}$ magnification and were merged together, producing a unique histologic image (Adobe Photoshop CS6 software, Adobe Systems Incorporated-Version 13.0x64, USA). A virtual section from the scanned ovary was chosen to match the histological section and compare the structures.

\section{RESULTS}

In all the seven species used, the images obtained by microCT scanning were remarkably similar to the reconstructed histologic sections, validating every finding. In the ovary of queens (Fig. 1a), the female donkey (Fig. 1b) and mares (Fig. 1c) high calibre blood vessels were concentrated in the medulla, branching into thinner vessels towards the cortex. In sows (Fig. 1d), cows (Fig. 1e) and rats (Fig. 1f) the medium calibre blood vessels were spread throughout the ovarian tissue. In the ovaries of the female donkey and (Fig. 1b) mares (Fig. 1c) the inverted position of cortical and medullary regions was clearly seen. In these two species, the medulla is the outer region of the ovary.

MicroCT allowed precise visualization of antral follicles and corpus luteum, confirmed by histology, in the ovaries from queens (Figs. 2a and 2b), female dogs (Figs. 2c and 2d) and sows (Figs. 2e and 2f), as well as in cows (Fig. 3), rats and mares. Additionally, microCT allowed a $3 \mathrm{D}$ reconstruction of the ovary permitting highresolution visualization of specific virtual sections in any chosen axis (Fig. 3). This tool even enabled the visualization of oocytes inside some antral follicles (Fig. 4) in this study.

The contrast tool from DataViewer software allowed the quality of the images to be increased, especially for the visualization of the corpus luteum (Fig. 5). Corpus luteum were more abundant than antral follicles in the ovaries of mares (Fig. 5a), sows (Fig. 5b) and rats (Fig. 5c), probably due to the oestral cycle stage of each animal at the moment of sampling.

Histology showed the presence of preantral follicles in the ovaries of queen, female dogs, rats, sows and cows, even though microCT did not allow the visualization of these structures. Nevertheless, the colour device from DataViewer allowed the identification of the region in which histology showed high number of preantral follicles in the queen's ovary (Fig. 6). In addition, this tool improved the cortex and medulla delimitation.

\section{DISCUSSION}

This is the first report using microCT to explore and compare ovarian structures in several domestic mammals. In all seven species, it was possible to distinguish the regions of cortex and medulla, identify antral follicles and corpus luteum and visualize the distribution of blood vessels. Nowadays, the main method for evaluation of the 

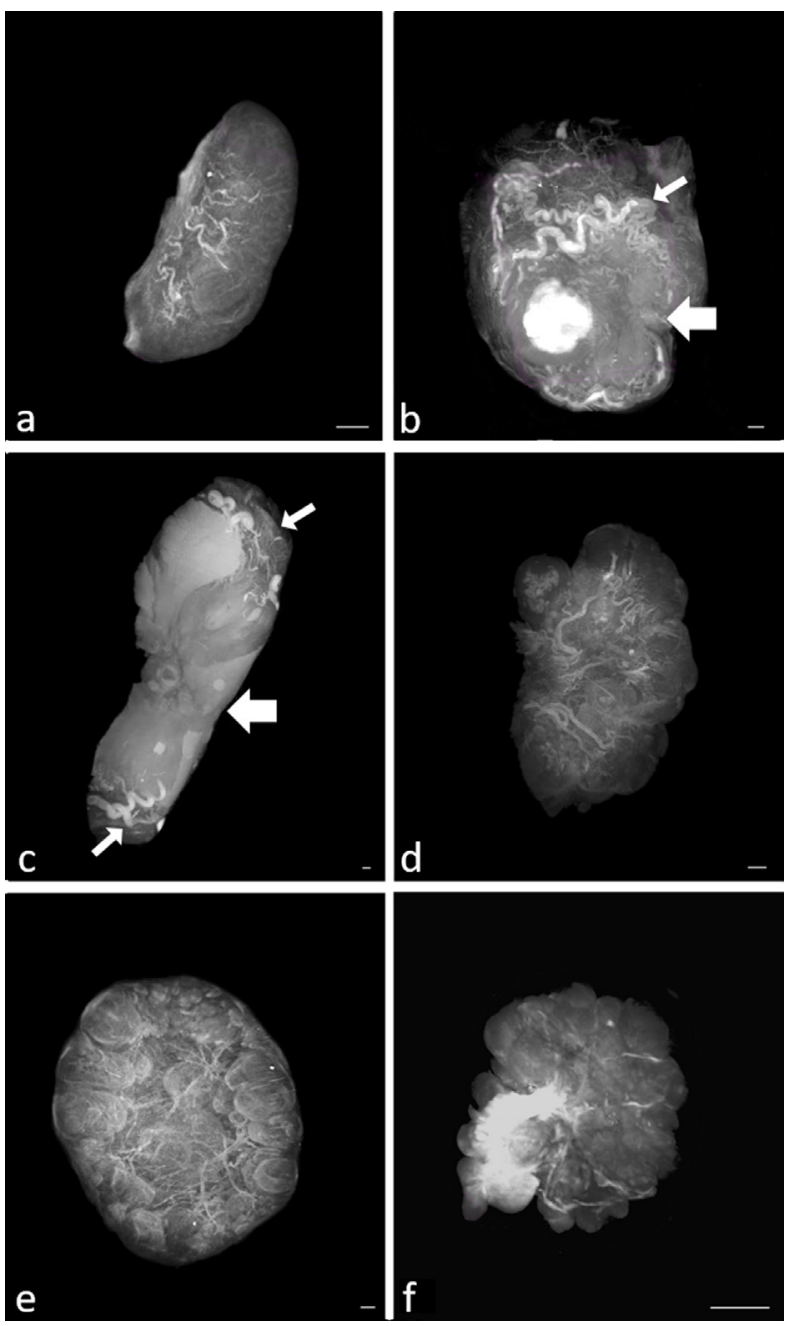

Figure 1- MicroCT images of the ovary with emphasis on blood vessels. Blood vessels are concentrated in the medullar region in the ovaries of queen (a), female donkey (b) and mare (c), while spread throughout the whole organ in sow (d), cow (e) and rat (f). Thin arrows: medullar region, thick arrows: cortex region. White areas reveal greater concentration of the contrast substance in $\mathbf{b}$ and $\mathbf{f}$. Barr $=2.5 \mathrm{~mm}$.

ovaries is transvaginal ultrasonography. However, transvaginal and/or transrectal ultrasonography is not viable in some smaller animals, such as rodents, small primates and even queens. The alternative use of transabdominal ultrasonography is limited to identifying and measuring antral follicles (Morgan et al. 1987). The precise visualization of the ovary by ultrasound is also difficult due to the overlap of pelvic bones, or the presence of faeces and gas (Jones et al. 2010). The results showed that microCT could be suitable for ovarian investigation in domestic mammals and may be a promising technique to explore ovarian structures, specially in research works that need monitoring the development of the ovarian structures in live animals during a certain period of time.

In this study, the distribution of blood vessels was different among species. Medium calibre blood vessels were distributed throughout the organ in the ovaries of cows, sows and rats, while, in queens, mares and female donkeys, the higher calibre blood vessels of ovaries were concentrated in the medulla with thinner vessels in the cortex. Moreover, in the ovaries of the female donkey and mares the localization of blood vessels proved the inverted position of cortical and medullary regions, which has been previously reported (Ginther 1992). MicroCT was shown to be applicable for morphologic evaluation of blood vessels of medium calibre, but the resolution provided by the imaging system is limited to the visualization of capillaries (Nebuloni et al. 2014). In this research, the use of a device from DataViewer software enabled better definition and improved images of blood vessels to be achieved. The contrast and colour tools of this software are dynamic and easy to use. Precise observation of blood vessels is important to identify the periovulatory period, since it is marked by intense neovascularization (Ginther et al. 2014). Doppler ultrasound enables the visualization of neovascularization, and it can be used to determine the fertile period in women (Vlaisavlevic and Došen 2007). Likewise, CT has been used for the evaluation of blood vessels in periovulatory stages in humans as well (Rozenblit et al. 2001, Saksouk and Johnson 2004). Meticulous in vivo investigation of the microvascular network is also necessary for other situations, such as in the differential diagnosis of malignant tumours, and CT seems appropriate for this type of analysis (Nebuloni et al. 2014). In fact, some researchers have used CT to identify 

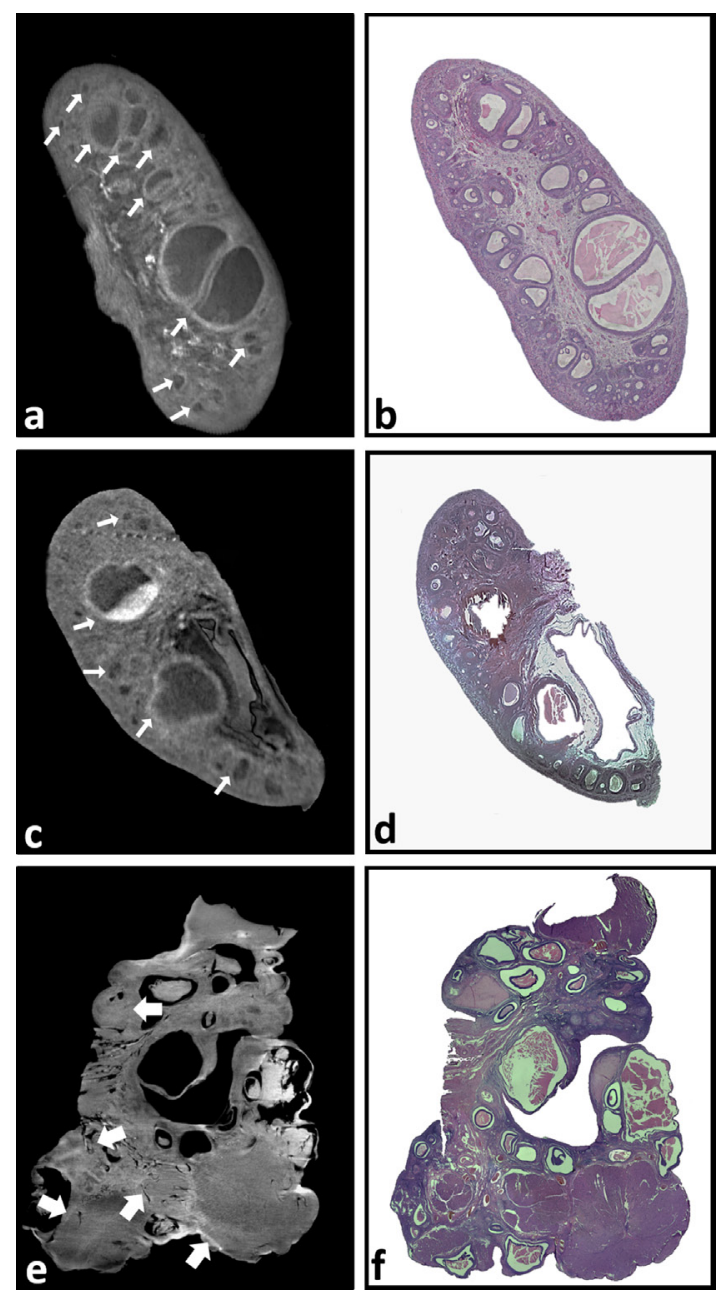

Figure 2 - MicroCT and histology images of the ovaries. $\mathbf{a} ; \mathbf{b}$ : queen $($ Barr $=2 \mathrm{~mm})$. $\mathbf{c}$; d: female dog $($ Barr $=$ $2 \mathrm{~mm})$. e; f: sow $($ Barr $=1.4 \mathrm{~mm})$. MicroCT images were chosen to match the histological section. Thin arrows: antral follicles, Thick arrows: corpus luteum. The structures observed in microCT were all confirmed by histology.

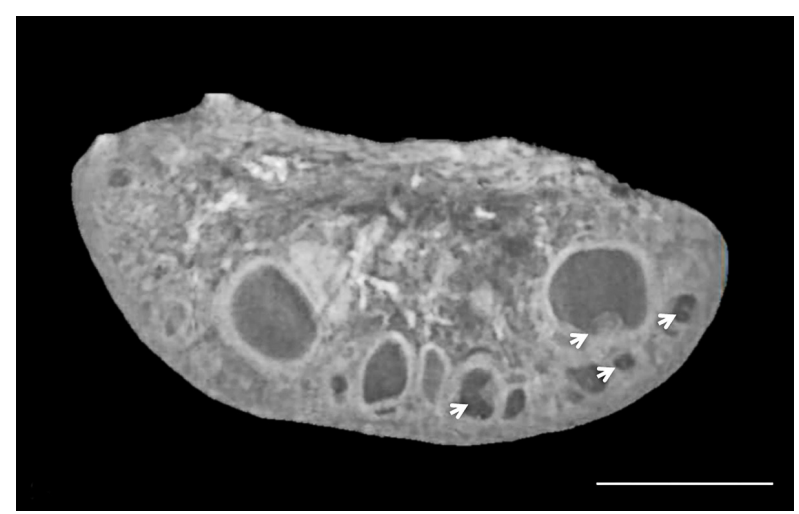

Figure 4 - MicroCT image showing oocytes inside antral follicles (white arrows) in the ovary of a queen. Barr $=2 \mathrm{~mm}$.
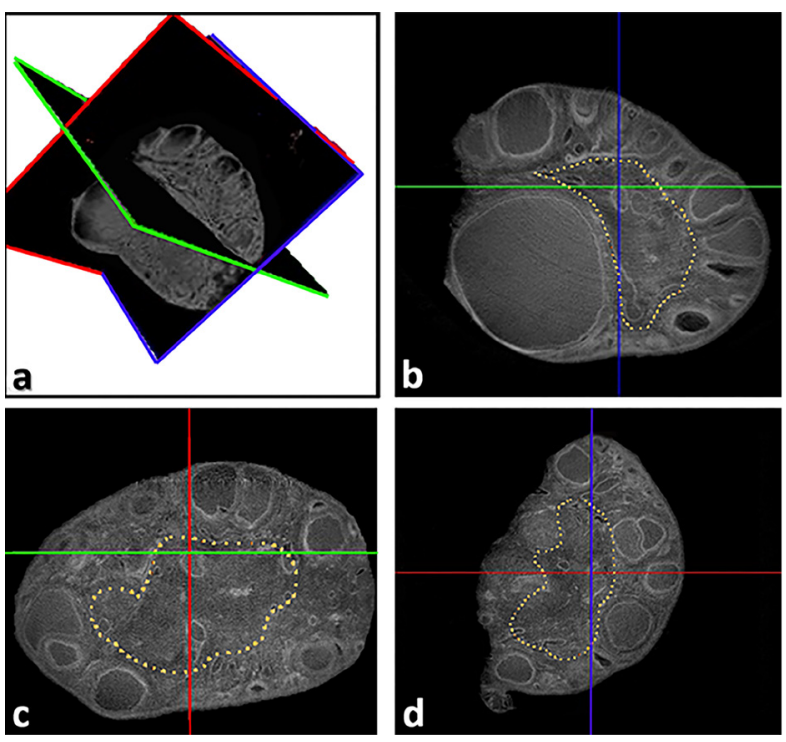

Figure 3 - Reconstructed microCT images of the cow ovary in $3 \mathrm{D}$ showing good definition of cortex, medulla and antral follicles. a: 3D image showing virtual sections of the three axis: sagittal (red), axial (blue) and coronal (green). b: Virtual section of the sagittal axis. c: Virtual section of the axial axis. d: Virtual section of the coronal axis. The medullar region is highlighted by a dotted line. Several antral follicles are present in the cortex.

ovarian tumours (Hamm et al. 1999, Tempany et al. 2000, Togashi 2003, Tsili et al. 2008, Perera and Prabhakar 2015) and even differentiate malignancy (Kurtz et al. 1999). MicroCT may be a valuable tool in researches concerning ovarian tumor using small animals models.

In all mammalian species studied, microCT was suitable for the observation of ovarian structures (i.e. corpus luteum and antral follicles). The images registered were confirmed by histology. Only one research team validated the $\mathrm{CT}$ findings with histology (Jones et al. 2007, 2010), using primate ovaries. Antral follicles were observed in those studies (Jones et al. 2007, 2010), as well as in ours, suggesting that $\mathrm{CT}$ could be applied in monitoring the oestral cycle and the diagnosis of some ovarian pathologies. In humans, the visualization of antral follicles with CT was used to diagnose cysts and polycystic ovaries (Foshager and Walsh 1994, Saksouk and Johnson 2004, Shaaban and Rezvani 

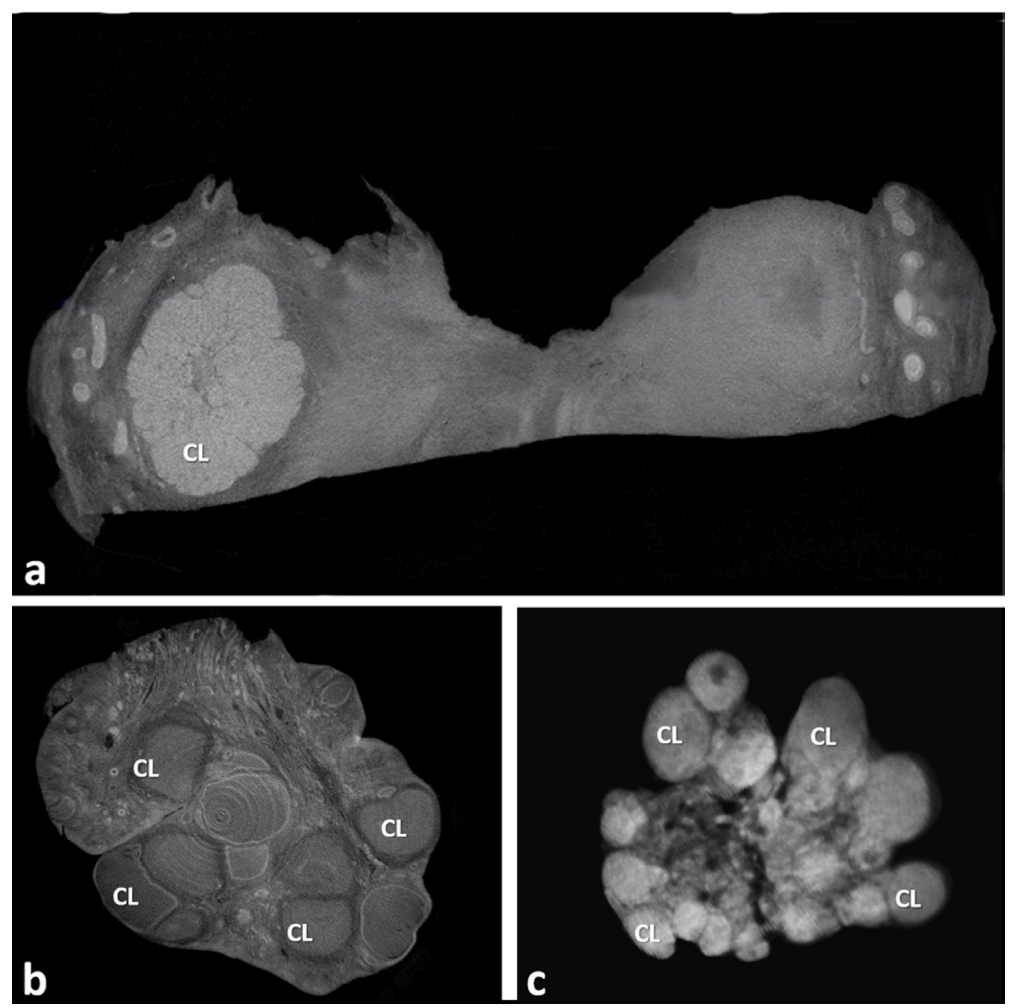

Figure 5 - MicroCT image obtained using the contrast tool (DataViewer software) that permits a better view of corpus luteum in the ovary. a: mare. b: sow. c: rat. CL: corpus luteum. Barr $=2.5 \mathrm{~mm}$.
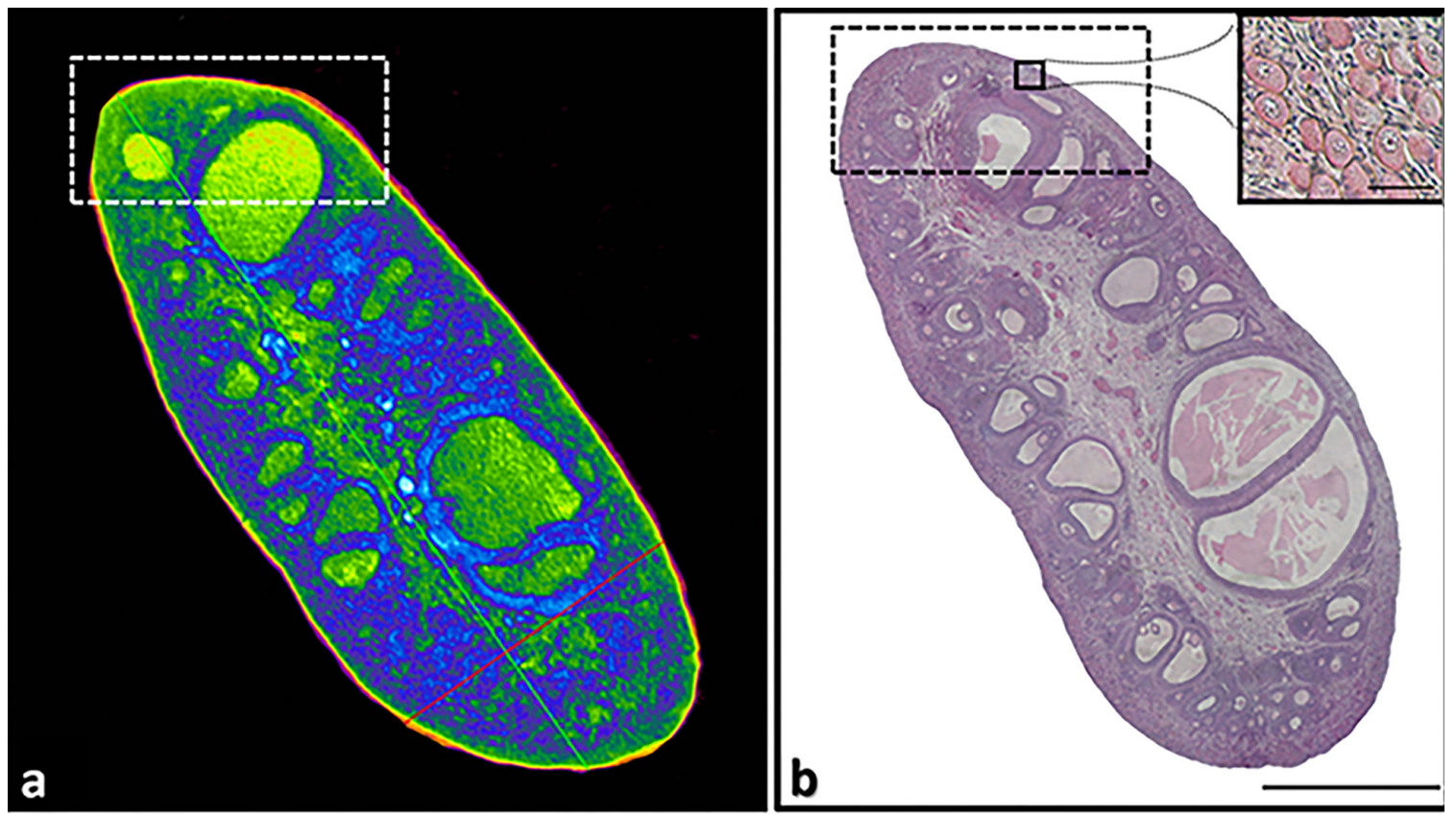

Figure 6 - MicroCT image using the color tool of the software DataViewer (a) and reconstructed histological section (b) of queen ovary (Barr $=2 \mathrm{~mm}$ ). The region with preantral follicle is highlighted in green in the microCT image (dotted box), as well as the medullar area and the antrum of antral follicles. Insert: Amplified region with many preantral follicles $($ Barr $=50 \mu \mathrm{m})$. 
2009). Corpus luteum was accurately seen as well. The identification of corpus luteum and albicans have been reported by other authors using CT (Rieber et al. 2001, Hariri et al. 2010). In contrast, follicles and corpus luteum were poorly identified using magnetic resonance imaging (Nöthling et al. 2006). Beyond the most commonly evaluated structures, in our study, oocytes could be observed within antral follicles. Thus, CT may complement or sometimes replace the ultrasonography diagnosis.

Although preantral follicles could not be adequately seen in the present study, probably because of their small size and the absence of liquid in these microstructures, the cortex area presenting a significant concentration of preantral follicles was observed in the queen's ovary with the use of DataViewer software. The use of the software highlighted preantral follicle regions, possibly due to its hypodense characteristic, as in the medullary region. Further studies are necessary to better investigate these findings, which might open interesting prospects for future applications of microCT.

Although we used surgically removed ovaries in this work, tomography can be used in vivo as well, as already carried out in humans (Foshager and Walsh 1994, Rozenblit et al. 2001). In this study, the ovaries were immersed in iodine solution, which provides good contrast to visualize different ovarian structures using microtomography. Contrasting substances are also used in vivo by intravenous injection, resulting in a good visualization of blood vessels and other structures. Contrast agents other than iodine may be used as well, such as iohexol and barium sulphate (Gregor et al. 2012). Alternatively, other contrast methods based in nanoparticles are being developed (Samei et al. 2009, Ashton et al. 2014).

The results of this research suggest the further use of $\mathrm{CT}$ as a non-invasive approach to study ovarian structures in small animals. This may be especially attractive for researches that need to follow the development of the ovarian structures and/or tumors in live animals. In those cases, invasive techniques usually require animals' euthanasia, not allowing the monitoring of the same animal throughout time, and the non-invasive techniques available, such as ultrasound, do not provide high spatial resolution. Of course, in vivo scanning brings up many additional challenges compared to ex vivo scanning, including exposure to radiation, which needs some consideration.

\section{CONCLUSIONS}

MicroCT presented great resolution to analyse ovarian structures, allowing perfect visualization of corpus luteum and antral follicles, even with the observation of oocytes. All tomography findings were confirmed by histology. Thus, this study draws attention to microCT as a promising technique for scientific study of development of ovarian structures and/or ovarian pathologies in small animals' models. We emphasize that further in vivo experiments will be necessary to check the feasibility of visualization of ovarian structures in live animals using microCT.

\section{ACKNOWLEDGMENTS}

The authors thank the Conselho Nacional de Desenvolvimento Científico e Tecnológico (CNPq), Coordenação de Aperfeiçoamento de Pessoal de Nível Superior (CAPES) and Financiadora de Estudos e Projetos (FINEP) for the financial support.

\section{REFERENCES}

ASHTON JR, CLARK DP, MODING EJ, GHAGHADA K, KIRSCH DG, WEST JL AND BADEA CT. 2014. DualEnergy Micro-CT Functional Imaging of Primary Lung Cancer in Mice using Gold and Iodine Nanoparticle Contrast Agents: A Validation Study. PLoS ONE 9: e88129.

BARRETO M, SCHOENHAGEN P, NAIR A, AMATANGELO S, MILITE M, OBUCHOWSKI N, 
LIEBER M AND HALLIBURTON S. 2008. Potential of dual-energy computed tomography to characterize atherosclerotic plaque: ex vivo assessment of human coronary arteries in comparison to histology. J Cardiovasc Comput Tomogr 2(4): 234-242.

BONTRAGER KL. 2003. Tratado de técnica radiológica e base anatômica. Rio de Janeiro: Guanabara-Koogan, 805 p.

DAVIS GR AND WONG FSL. 1996. X-ray microtomography of bones and teeth. Physiol Meas 17(3): 121-146.

FOSHAGER MC AND WALSH JW. 1994. CT anatomy of the female pelvis: a second look. Radiographics 14(1): 51-64.

GINTHER OJ. 1992. Reproductive biology of the mare: basic and applied aspects, $2^{\text {nd }}$ ed., Cross Plains: Equiservices Publishing, $642 \mathrm{p}$.

GINTHER OJ, RAKESH HB AND HOFFMAN MM. 2014. Blood flow to follicles and CL during development of the periovulatory follicular wave in heifers. Theriogenology 82(2): 304-311.

GIORDANO LA, GIORDANO MV AND SILVA RO. 2009. Tumores anexiais na adolescência. Adolesc Saude 6(4): 48-52.

GREGOR T ET AL. 2012. Correlating Micro-CT imaging with quantitative histology. In: Injury and Skeletal Biomechanics. Croatia: Intech, p. 173-196.

GULDBERG RE ET AL. 2003. Analyzing bone, blood vessels, and biomaterials with microcomputed tomography. IEEE Eng Med Biol Mag 22(5): 77-83.

HAMM B. 1994. Computerized tomography and MR tomography in diagnosis of ovarian tumors. Radiology 34(7): 362-369.

HAMM B, KUBIK-HUCH RA AND FLEIGE B. 1999. MR imaging and $\mathrm{CT}$ of the female pelvis: radiologic-pathologic correlation. Eur Radiol 9(1): 3-15.

HARIRI LP, LIEBMANN ER, MARION SLL, HOYER PB, DAVIS JR, BREWER MA AND BARTON JK. 2010. Simultaneous optical coherence tomography and laser induced fluorescence imaging in rat model of ovarian carcinogenesis. Cancer Biol Ther 10(5): 438-447.

JONES JC, APPT SE, BOURLAND JD, HOYER PB, CLARKSON TB AND KAPLAN JR. 2007. Multidetector computed tomographic morphology of ovaries in cynomolgus macaques (Macaca fascicularis). J Am Assoc Lab Anim 46(5): 54-63.

JONES JC, APPT SE, WERRE SR, TAN JC AND KAPLAN

JR. 2010. Validation of multi-detector computed tomography as a non-invasive method for measuring ovarian volume in macaques (Macaca fascicularis). Am J Primatol 72(6): 530-538.

KURTZ AB ET AL. 1999. Diagnosis and staging of ovarian cancer: comparative values of Doppler and conventional US, CT, and MR imaging correlated with surgery and histopathologic analysis-report of the Radiology Diagnostic Oncology Group. Radiology 212(1): 19-27.
LANGHEINRICH A, BOHLE R, GRESCHUS S, HACKSTEIN G, VON GERLACH S, RAU W AND HOELSCHERMANN H. 2004. Atherosclerotic lesions at micro-CT: feasibility for analysis of coronary artery wall in autopsy specimens. Radiology 231(3): 675-681.

LANGHEINRICH AC, YENIGUEN M, OSTENDORF A, MARHOFFER S, KAMPSCHULTE M, BACHMANN G, STOLZ E AND GERRIETS T. 2010. Evaluation of the middle cerebral artery occlusion techniques in the rat by in-vitro 3-dimensional micro- and nano computed tomography. BMC Neurol 10: 36.

METSCHER BD. 2009a. MicroCT for developmental biology: a versatile tool for high-contrast 3D imaging at histological resolutions. Dev Dynam 238(3): 632-640.

METSCHER BD. 2009b. MicroCT for comparative morphology: simple staining methods allow high-contrast 3D imaging of diverse non-mineralized animal tissues. BMC Physiology 9: 11.

MORGAN PM, HUTZ RJ, KRAUS EM, CORMIE JA, DIERSCHKE DJ AND BAVISTER BD. 1987. Evaluation of ultrasonography for monitoring follicular growth in rhesus monkeys. Theriogenology 27(5): 769-780.

MORIBATA Y, KIDO A, YAMAOKA T, MIKAMI Y, HIMOTO Y, KATAOKA M, FUJIMOTO K, KONISHI I AND TOGASHI K. 2015. MR imaging findings of ovarian torsion correlate with pathological hemorrhagic infarction. J Obstet Gynaecol Res 41(9): 1433-1439.

NEBULONI L, KUHN GA, VOGEL J AND MULLER R. 2014. A novel in vivo vascular imaging approach for hierarchical quantification of vasculature using contrast enhanced Micro-Computed Tomography. PLoS ONE 9(1): e86562.

NÖTHLING JO, DE CRAMER KG, GERBER D AND KAMMER VR. 2006. Luteal and follicular count in bitches: assessment by means of magnetic resonance imaging. Theriogenology 66(5): 1343-1354.

PERERA DS AND PRABHAKAR HB. 2015. Imaging of the Adnexal Mass. Clin Obstet Gynecology 58: 28-46.

POSTNOV A, MEURRENS K, WEILER H, VANDYCKD XUH, TERPSTRA P AND DE CLERCK NM. 2005. In vivo assessment of emphysema in mice by high resolution X-ray microtomography. J Microsc 220: 70-75.

PRATER PE, SHIRES M AND COLEY RB. 1988. Diagnostic aids for ovarian neoplasia: serologic interpretation and ultrasonography. Vet Med 83(12): 1273-1276.

RIEBER A, NUSSLE K, STOHR I, GRAB D, FENCHEL S, KREIENBERG R, RESKE SN AND BRAMBS HJ. 2001. Preoperative diagnosis of ovarian tumors with MR imaging: Comparison with transvaginal sonography, positron emission tomography, and histologic findings. AJR Am J Roentgenol 177(1): 123-129.

ROZENBLIT AM, RICCI ZJ, TUVIA J AND AMIS JR ES. 2001. Incompetent and dilated ovarian veins: a common 
CT finding in asymptomatic parous women. Am J Roentgenol 176(1): 119-122.

SAKSOUK FA AND JOHNSON SC. 2004. Recognition of the ovaries and ovarian origin of pelvic masses with CT. Radiographics 24: S133-S146.

SAMEI E, SAUNDERS RS, BADEA CT, GHAGHADA KB, HEDLUND LW, QI Y, YUAN H, BENTLEY RC AND MUKUNDAN JR S. 2009. Micro-CT imaging of breast tumors in rodents using a liposomal, nanoparticle contrast agent. Int J Nanomed 4: 277-282.

SAWYER RW, VICK CW, WALSH JW AND MCCLURE PH. 1985. Computed tomography of benign ovarian masses. J Comput Assist Tomogr 9(4): 784-789.

SHAABAN A AND REZVANI M. 2009. Ovarian cancer: detection and radiologic staging. Clin Obstet Gynecol 52(1): 73-93.
TEMPANY CM, ZOU KH, SILVERMAN SG, BROWN DL, KURTZ AB AND MCNEIL BJ. 2000. Staging of advanced ovarian cancer: comparison of imaging modalitiesreport from the radiological diagnostic oncology group. Radiology 215(3): 761-767.

TOGASHI K. 2003. Ovarian cancer: the clinical role of US, CT, and MRI. Eur Radiol 13(4): L87-104.

TSILI AC, TSAMPOULAS C, ARGYROPOULOU M, NAVROZOGLOU I, ALAMANOS Y, PARASKEVAIDIS E AND EFREMIDIS SC. 2008. Comparative evaluation of multidetector CT and MR imaging in the differentiation of adnexal masses. Eur Radiol 18(5): 1049-1057.

VLAISAVLJEVIC V AND DOŠEN M. 2007. Clinical applications of ultrasound in assessment of follicle development and growth. DSJUOG 1(2): 50-63. 